Indian J. Anim. Hlth. (2020), 59(1) : 50-54

DOI: $10.36062 /$ ijah.59.1.2020.50-54

\title{
PATTERN OF ANTIBIOTICS USE IN PIGS OF NORTH EASTERN INDIA
}

\author{
S. KUMAR ${ }^{1}$, J. BHARATI ${ }^{1}$, A. K. YADAV ${ }^{1}$, S. R. PEGU ${ }^{1}$, J. DOLEY ${ }^{1}$, \\ S. PAUL ${ }^{1}$, B. C. DAS ${ }^{1}$, N. H. MOHAN ${ }^{1}$ AND R. DEB ${ }^{1 *}$ \\ ${ }^{1}$ ICAR-National Research Center on Pig, Rani-781 131, Guwahati, Assam, India
}

Pig farming is an important way of livelihood for people of North East India. Therapeutic and prophylactic use of antibiotics on pig farms has become an integral part of farm health management. This practice has raised concerns of antimicrobial resistance (AMR) in animals, and moreover, the consumers. AMR is selected and perpetuated not only by the regular use of antibiotics as therapeutic agents, but also as feed additives in animals. In the present study a survey was conducted in six pig farms of three NE states of India regarding the use of antibiotics in feed and treatment purpose. It was observed that penicillin, ampicillin, cephalosporin, cephalaxin and clavulanic acid plus amoxycillin were commonly used antibiotics for the therapeutic purpose. Monensin, amprolium, bacitracin, neomycin and chlortetracycline were used in the feed for prophylaxis, and amoxicillin, oxytetracycline and streptomycin were used for dual purpose. Antibiotics as a growth promoter, improves growth rate, feed efficiency, reduce mortality and morbidity, and improves reproductive performance. The observations of the present study may provide an idea of quantitative contribution of pig farming to antimicrobial resistance and can help in building appropriate intervention strategies to optimize the use of antibiotics in pig production.

Key words: Antibiotics, Antimicrobial resistance, Prophylaxis, Therapeutics

Pig farming is an important source of livelihood for the poor and tribal people of North Eastern (NE) India (Haldar et al., 2017). It improves the livelihood and food security of the poor people by providing protein with essential amino acids and income (Chauhan et al., 2016). India has a total 9.06 million pig population of which $46.88 \%$ is located in eight NE states. Assam has the highest pig population in India followed by Jharkhand, Meghalaya and West Bengal (Basic Animal Husbandry Statistics2019, DAHD, GOI). Antibiotics and antimicrobial agents are the major therapeutic options to treat the animals and protect them from various infectious diseases. Beside treatment, antibiotics are also used as feed additive to improve the growth rate, reproductive and lactation performances, reduce subclinical diseases, mortality and morbidity, and overall health condition in pigs (Cromwell, 2002). The rampant use of antibiotics in animals as growth promoters, metaphylaxis and prophylaxis remain a major driver in development of antibiotic resistance due to selection pressure. Antibiotics were mainly used to improve average daily gain in weight, decrease morbidity and mortality (Chattopadhyay, 2014). Rising global concern about AMR has drawn attention to the use of antibiotics in livestock. Understanding the current 
usage of antibiotics in food animals, particularly in pigs is essential for effective interventions in optimisation of antibiotic use. In spite of the concern about the relationship between the use of antibiotics and AMR in food animals like pig and AMR in humans, there are limited information available from the low- and middle-income countries which describe the antibiotics usage pattern in piggery sector and the factors that influence the farmers to use them (Lekagul et al., 2020). In order to design and implement effective interventions that will reduce the unnecessary use of antibiotics in livestock, an understanding of current usage is essential. In India there is lack of study describing the pattern of antibiotic use in piggery sector. The present study was formulated to explore the types of antibiotics used by pig farms and the pattern of use of antibiotics in pigs in NE region of India.

\section{MATERIALS AND METHODS}

To know the pattern of antibiotic use in the pig farm, data on the use of antibiotics in the therapeutics and in the feed were collected through an online farm interview questionnaire with the help of Veterinary officers and animal health workers from three pig farms in Arunachal Pradesh, two pig farms in Meghalaya and one farm in Assam randomly. Details of the different variables considered for the present study is depoicted in Table 1. A questionnaire is prepared to collect information on location, herd size, number of animals, health status of the animals, prevalence of common diseases, vaccination status of the animals and types of antibiotics commonly used for treatment, prophylaxis and growth promotion.

\section{RESULTS}

It is observed that penicillin, ampicillin, cephalosporin, cephalaxin and amoxicillin plus clavulanic acid are commonly used antibiotics for the therapeutic purpose in all the three states. Antibiotics used in the feed as prophylaxis are monensin, amprolium, bacitracin, neomycin and chlortetracycline. Antibiotics used for dual purpose are amoxicillin, oxytetracycline and streptomycin (Table 2).

\section{DISCUSSION}

Present study focused on use pattern of antibiotics in pig farms in and around North Eastern region

Table 1. Variables used as parameters for the questionnaire based survey

\begin{tabular}{lllllll}
\hline Variables & \multicolumn{3}{c}{ Arunachal Pradesh } & \multicolumn{2}{c}{ Meghalaya } & Assam \\
\cline { 2 - 7 } & Farm 1 & Farm 2 & Farm 3 & Farm 4 & Farm 5 & Farm 6 \\
\hline Herd Size & Small & Small & Small & Medium & Small & Small \\
Number of animals & 145 & 160 & 130 & 215 & 85 & 80 \\
Type of Farm & Unorga- & Unorga- & Unorga- & Unorga- & Unorga- & Unorga- \\
(Organized / unorganized) & nized & nized & nized & nized & nized & nized \\
Vaccination (Commonly & Yes & Yes & Yes & Yes & Yes & Yes \\
given vaccines like Swine & & & & & & \\
$\begin{array}{l}\text { Fever, FMD etc.) } \\
\text { Veterinary aid availability }\end{array}$ & Yes & Yes & Yes & Yes & Yes & Yes \\
Mortality of pigs (Age) & $1-3$ & 1 months & Above 3 & Above 3 & $1-3$ & $1-3$ \\
& months & & months & months & months & months \\
Prevalence of common & Diarrhea, & Diarrhea, & Fever, & Diarrhea, & Diarrhea, & Diarrhea \\
diseases & Fever, & weakness & Skin & Fever & Skin & \\
& Skin & & diseases, & & infection & \\
& infection & & Diarrhea & & & \\
\hline
\end{tabular}




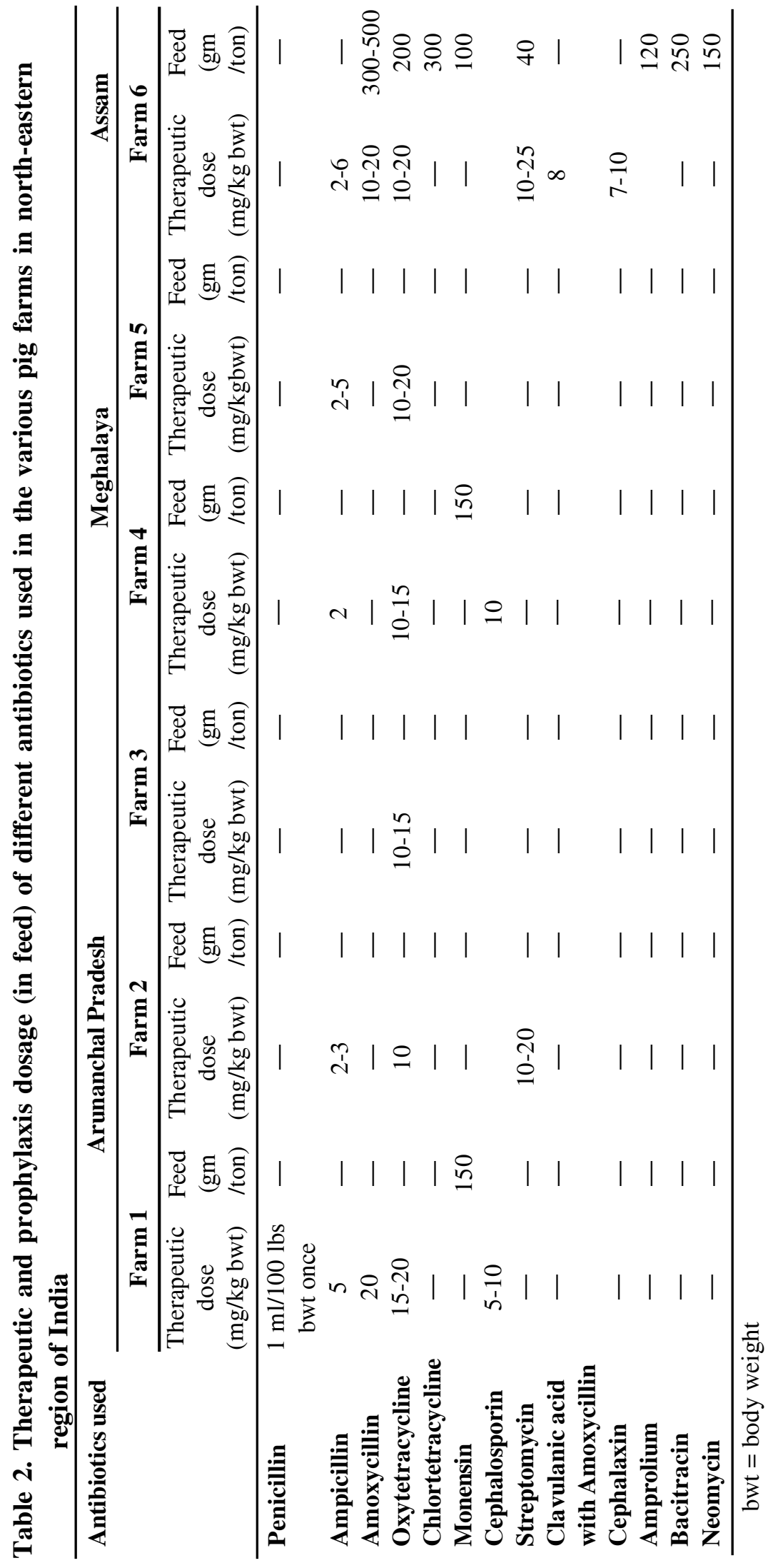


of India. Similar to our finding, Lekagul et al. (2019) also found that penicillins and tetracyclines were the most commonly used in pig farms of many countries including China, Brazil, Sweden, Germany and Canada. Antibiotics were more frequently administered through feed than through water in all the states under study.

Majority of the antibiotics commonly used in pig farms are categorised as critically important antimicrobials (CIA) for treating humans (WHO CIA list). The WHO CIA list categorizes medically important antimicrobials into three categories: important, highly important and critically important, and further divides the last category into 'high priority' CIA including aminoglycosides, aminopenicillins and carbapenems; and 'highest priority' CIA including cephalosporins (third, fourth and fifth generation), glycopeptides, macrolides, polymyxin (colistin) and quinolones (WHO, $2019 a ; 2019 b)$. Nearly half of the oral and injectable antibiotics used in farms and twothirds of antibiotics added in medicated feed belonged to the CIA category. In our study, high priority CIA streptomycin, amoxycillin and neomycin were used as feed additive and for therapeutics, whereas ampicillin was used only for therapeutics. Highest priority CIA cephalexin was used only for therapeutic purpose.

The use of antibiotics in pigs is complex and associated with the interrelating domains of animal health, animal welfare and economics. Antibiotics have been used routinely in farm animal production since the 1950s to treat, control and prevent disease and to increase productivity. Based on the predicted continued rise in global demand for livestock products, global antimicrobial consumption of livestock is predicted to increase by two-thirds over the next 10 years. Within the livestock sector, antimicrobial consumption is estimated to be highest in pigs, compared with chicken and cattle (van Boeckel et al., 2015).

Subtherapeutic doses of antibiotics in foodanimals has been a common practice for decades for a number of reasons like control of the spread of symptomatic infections between animals in close contact, some of which may be subclinically infected; to prevent disease at points of high risk prior to the onset of symptoms, particularly when animals are under stress (e.g. extreme weather, post vaccination or moving pen) and to improve production performance (Landers et al., 2012). In pigs, antibiotics are applied to whole groups by mixing antibiotics with feed (medicated feed) or adding antibiotic powder or solution into drinking water (medicated water) (van Rennings et al., 2015).

A number of studies have reported that rampant use of antibiotics can seriously impact an animal's well-being and can also result in increased antibiotic usage due to recurrence and spread of infection due to AMR. Indeed there is emerging evidence of the threat including a recent report describing the discovery of a plasmid-mediated colistin-resistant gene in commensal Escherichia coli from tests on pigs, pork products and humans in China (Liu et al., 2016). Antibiotic resistance in bacteria is affected by the type of antibiotics used and how they are handled. Therefore proper assessment of the clinical case is very critical, in deciding the type and dose of antibiotics.

Analyzing antibiotics use pattern can help to identify scenarios where use is higher or unnecessary and from there a manageable solution to reduce application of antibiotics in that particular area of production can be developed. A better understanding of antibiotic use in pigs, particularly in pork consuming regions, can help design appropriate intervention strategies to optimize the use of antibiotics in pig production (Moreno, 2012). If the primary causes of disease, mortality and antibiotic use are addressed at a pig farm, the usage of antibiotics can be reduced substantially. Farm management practices that can maximize natural immunity and minimize stress can reduce the significant use of antibiotics, since decreased immunity and increased stress is linked to increased incidences of diseases (Postma et al., 2015). Alternative approaches need to be sought 
to maintain herd health and productivity in order to protect the effectiveness of antibiotics. These solutions need to be tested and demonstrated to pig farmers to show their relative costeffectiveness. A progressive restriction in the use of antibiotics in pigs with an emphasis on CIA as advocated earlier, is the need of the hour. This can be achieved by controlling the distribution of certain antibiotics for animal use with medicines available only on prescription. The government should strengthen veterinary services to improve access of farmers to animal health advice and explore alternative

\section{REFERENCES}

Chattopadhyay MK, 2014. Use of antibiotics as feed additives: A burning question. Front Microbiol, 5: 334

Chauhan A, Patel BHM, Maurya R, Kumar S, Shukla S et al., 2016. Pig production system as a source of livelihood in Indian scenario: An overview. Int J Sci Environ Technol, 5(4): 2089-2096

Cromwell GL, 2002. Why and how antibiotics are used in swine production. Anim Biotechnol, 13(1): 7-27

Haldar A, Das D, Santra A, Pal P, Dey S et al., 2017. Traditional feeding system for pigs in northeast India. Int J Livest Res, 7(8): 122-132

Landers TF, Cohen B, Wittum TE and Larson EL, 2012. A review of antibiotic use in food animals: perspective, policy, and potential. Public Health Rep, 127(1): 4-22

Lekagul A, Tangcharoensathien V and Yeung S, 2019. Patterns of antibiotic use in global pig production: A systematic review. Vet Anim Sc, 7: 100058

Lekagul A, Tangcharoensathien V, Mills A, Rushton J and Yeung S, 2020. How antibiotics are used in pig farming: A mixed-methods study of pig farmers, feed mills and veterinarians in Thailand. BMJ Global Health, 5(2): e001918

Liu YY, Wang Y, Walsh TR, Yi LX, Zhang R et al., 2016. Emergence of plasmid-mediated colistin resistance mechanism MCR-1 in animals and human beings in China: A microbiological and molecular biological study. Lancet Infect interventions. Progressive restriction in the use of antibiotics in pigs through using prescriptions to control the distribution of certain antibiotics should be the norm. Though the present study is limited by the sample size and diversity of farms, yet they are indicative of antibiotic use pattern.

Knowledge on antibiotic use and AMR awareness among the pig farmers and veterinarians can help alleviate this problem and a systematic approach should be developed to address the overuse of antibiotics keeping into account both animal and human welfare.

Dis, 16(2): 161-168, doi:10.1016/S14733099(15)00424-7

Moreno MA, 2012. Survey of quantitative antimicrobial consumption in two different pig finishing systems. Vet Rec, 171(13): 325

Postma M, Stärk KDC, Sjölund M, Backhans A, Beilage EG et al., 2015. Alternatives to the use of antimicrobial agents in pig production: A multi-country expert-ranking of perceived effectiveness, feasibility and return on investment. Prev Vet Med, 118(4): 457-466

van Boeckel TP, Brower C, Gilbert M, Grenfell BT, Levin SA et al., 2015. Global trends in antimicrobial use in food animals. Proc Natl Acad Sci USA, 112(18): 5649-5654, doi: 10.1073/pnas. 1503141112

van Rennings L, von Münchhausen C, Ottilie H, Hartmann M, Merle R et al., 2015. Cross-sectional study on antibiotic usage in pigs in Germany. PLoS One, 10(3): e0119114, doi: 10.1371/journal.pone.0119114

WHO, 2019a. World Health Organization. Critically important antimicrobials WHO CIA list. Geneva. https://www.who.int/foodsafety/areas_work/ antimicrobial-resistance/cia/en/[Accessed 06 June 2020]

WHO, 2019b. World Health Organization. List of critically important antimicrobials for human medicine (WHO CIA list $6^{\text {th }}$ revision), https:// www.who.int/foodsafety/publications/ antimicrobials-sixth/en/ [Accessed 05 June 2020]

Received - 02.04.2020, Accepted - 21.05.2020, Published - 01.06.2020 